\title{
Anemia infantil: más allá del hierro oral
}

\author{
Childhood anemia: beyond oral iron
}

\author{
Víctor Hugo, Díaz-Silva ${ }^{1 a}$
}

\section{Sr. Editor:}

La prevalencia de anemia en los niños menores de tres años durante los últimos cinco años ha sido elevada en el Perú. El reporte de la Encuesta Familiar y Demográfica (ENDES) del año 2018 indica que la frecuencia de anemia infantil es 43,5\%; valor que ha disminuido levemente en los últimos cinco años. En la región Lambayeque, la situación es parecida ${ }^{(1)}$. La anemia infantil aún es un problema de salud nacional.

El Estado Peruano ha definido políticas de salud que buscan disminuir estos indicadores; sin embargo, los resultados no son los esperados. El "Plan Multisectorial de Lucha contra la Anemia" incluye, dentro de otros aspectos, la distribución de micronutrientes (MNN) y suplementos de hierro en todos los centros de atención primaria ${ }^{(2)}$. Lamentablemente, la ingesta de hierro en forma de MNN no ha logrado disminuir la prevalencia de anemia.

El incentivo del consumo de hierro no debe considerarse como el pilar del programa de prevención de la anemia infantil. Un estudio realizado en dos comunidades rurales de nuestro país encuentra que la frecuencia de anemia por déficit de hierro es menor al $25 \%{ }^{(3)}$. Existen otras causas de anemia infantil, tales como: deficiencia de vitamina B12, enfermedades genéticas, infecciosas o neoplasias hematológicas; aunque su frecuencia aún no ha sido estudiada en nuestro medio. El empleo de MNN en comunidades rurales evidencia un incremento no mayor a $1 \mathrm{~g} / \mathrm{dl}$ de hemoglobina ${ }^{(4)}$. En tal sentido, no todas las anemias son ferropénicas ni un incremento del valor de hemoglobina asegura mejorar las reservas de hierro. Por lo tanto, aumentar el consumo de hierro no asegura que los parámetros hematimétricos se mejoren sostenidamente.

Existen modelos de evaluación de los factores de riesgo de anemia por déficit de hierro. Los modelos propuestos en otros países sugieren mejorar el acceso al centro de salud comunitario, mejorar las condiciones sociales y económicas de las familias, fortalecer el vínculo madre-niño y adecuar las políticas de salud al contexto cultural de las comunidades ${ }^{(5)}$. La lucha contra la anemia infantil tiene más frentes que abordar; no debería centrarse en el consumo de hierro.

Finalmente, la anemia en niños menores de 36 meses no es necesariamente por deficiencia de hierro y el fomento de la ingesta de hierro, sea en forma de MNN o de alimentos fortalecidos o alimentos ricos en hierro, no logrará disminuir las altas cifras en nuestro país hasta que no se haya determinado previamente la verdadera frecuencia de anemia por déficit de hierro.

\footnotetext{
1. Departamento de área clínica, Hospital Regional Lambayeque, Chiclayo, Perú.

a. Médico cirujano especialista en Hematología. Maestro en Medicina.
} 


\section{REFERENCIAS BIBLIOGRÁFICAS}

1. Instituto Nacional de Estadística e Informática. Perú: Encuesta Demográfica y de Salud Familiar 2018. Lima: Instituto Nacional de Estadística e Informática; 2018: 244-54.

2. Ministerio de Desarrollo e Inclusión Social. Plan multisectorial de la lucha contra la anemia [Internet]. Lima. 124 p. Disponible en: http://www.midis. gob.pe/dmdocuments/plan-multisectorial-de-lucha-contra-la-anemia-v3.pdf.

3. Gonzales E, Huamán-Espino L, Gutiérrez C, Aparco JP, Pillaca J. Caracterización de la anemia en niños menores de cinco años de zonas urbanas de Huancavelica y Ucayali en el Perú. Rev Peru Med Exp Salud Publica. 2015; 32(15):431-9.

4. Munayco CV, Ulloa-Rea ME, Medina-Osis J, Lozano-Revollar CR, Tejada $\mathrm{V}$, Castro-Salazar C. Evaluación del impacto de los multimicronutrientes en polvo sobre la anemia infantil en tres regiones andinas del Perú. Rev Peru Med Exp Salud Publica. 2013; 30(2):229-34.

5. Falivene M, Fattore G. Abordaje multidimensional de la anemia por de ciencia de hierro en niños menores de dos años de edad del Noreste Argentino. Años 2004-2005. Arch Argent Pediatr. 2016; 114(1):14-22.

Revisión de pares: Recibido: 21/12/2019 Aceptado: 08/01/2020 